\title{
Evaluation Based on TCAD Simulation of Failure Tolerance of the Elements on the Cells STG DICE for 65-nm CMOS Blocks of Associative Memory
}

\author{
Yu.V. Katunin ${ }^{1}$, V.Ya. Stenin ${ }^{1,2}$ \\ ${ }^{1}$ Scientific Research Institute of System Analysis of RAS, Moscow, katunin@cs.niisi.ras.ru \\ ${ }^{2}$ National Research Nuclear University MEPhI, vystenin@mephi.ru
}

\begin{abstract}
The paper presents TCAD simulation of the $65 \mathrm{~nm}$ bulk CMOS logical element of matching, and element of masking based on STG DICE cell with the combinational logic. These items are used for the content addressable memory (associative memory) and the translation lookaside buffers for 65-nm CMOS bulk technology. The combinational logic consists of two tristate inverters (for elements of matching) and additionally consists of two normal inverters (for elements of masking). The STG DICE cell (Spaced Transistor Groups DICE) is different from the standard DICE (Double Interlocked Cell) in that the transistors are separated into two special groups. That is why the charge collection with the tracks of single nuclear particles impacted on one of these groups does not lead to upset of logical state of this memory cell. Failure tolerance of the elements of matching and elements of masking based on the STG DICE cell increases by the separation of its transistors (including the combination logic) into two blocks. Each block consists of one group of transistors of the STG DICE cell and transistors of one of two tristate inverters only for elements of matching and additionally one normal inverter for elements of masking. The charge collection with tracks of nuclear particles was simulated using tracks along the normal to the chip surface. Linear energy transfers were in range 10-60 $\mathrm{MeV} \times \mathrm{cm}^{2} / \mathrm{mg}$. The charge collection with tracks onto the linear energy transfer up to $60 \mathrm{MeV} \times \mathrm{cm}^{2} / \mathrm{mg}$ does not upset data in STG DICE cells with tracks along the normal to the chip surface. Pulses of error in the output of the combinational logic can occur up to duration $0.3-0.6 \mathrm{~ns}$ in the range of the energy transfer $30-60 \mathrm{MeV} \times \mathrm{cm}^{2} / \mathrm{mg}$.
\end{abstract}

Keywords - logical element, simulation, error impulse correction, nuclear particle, failure tolerance, track, memory cell.

\section{INTRODUCTION}

Matching in content addressable memory (CAM) is carried out today by logic elements on 6-transistor CMOS cells and Exclusive OR (XOR) combinational logic [1]. An important usage of associative memory are highdensity systems on the chip [2]. The base of these systems are the XOR Associative Memory cells (XORAM cells) on 6-transistor CMOS cells as proposed in [3].

Work continues on the joint use of correcting codes and the mutual topological arrangement of cells on the chip itself [4] in order to reduce the probability of multi- ple upsets. There are examples of the development of ternary-cells with increased failure resistance [5], [6], which did not become successful, because they were based on the modification of the traditional $6 \mathrm{~T}$ memory cells by the introduction of additional elements and connections. The reliability of 6-transistor memory cells under exposure to single nuclear particles has significantly deteriorated with the design rules reduced to 65-28 nm for the bulk CMOS technology. The resistance to upsets of the traditional DICE cells is the same as the CMOS 6T cells for the design rules below 65-28 $\mathrm{nm}$.

In previous works, we proved the separation of the transistors of the standard DICE cell into two groups [7] so that the particle impact on only one group does not cause the upset of the logical state of the cell [8]. This design of the STG DICE cell (Spaced Transistor Groups DICE) differs from that of a standard DICE cell [9].

Two groups are connected by two wires only, which saves silicon in case of increasing the distance between groups of one cell, using alternating groups of different cells. The maximum number of memory cells STG DICE with alternating groups of transistors is limited by the equality of the areas of the metallization layer of connections of the groups and the transistor layer [10]. Therefore, it is possible to increase the distances between mutually sensitive pairs of transistors from two groups without increasing the area practically. The distance between mutually sensitive nodes of two groups more than $2.0 \mu \mathrm{m}$ provides the highest fault tolerance of STG DICE cells. The experimental study demonstrated the high insensitivity of the 65-nm CMOS STG DICE to upsets [11].

The basic elements that can ensure the fault tolerance of associative memory to the ionizing effects of single nuclear particles are the elements of matching based on the STG DICE cell. Our recent TCAD simulation confirms [12] that the STG DICE cells can preserve their logical states when exposed to single nuclear particles with linear energy transfers (LETs) of up to $60 \mathrm{MeV} \cdot \mathrm{cm}^{2} / \mathrm{mg}$. The objective of the paper is studying new logical elements on the base of STG DICE cells to make sure it is possible to improve characteristics of content addressable memories designed to operate under the impacts of single ionizing nuclear particles. 


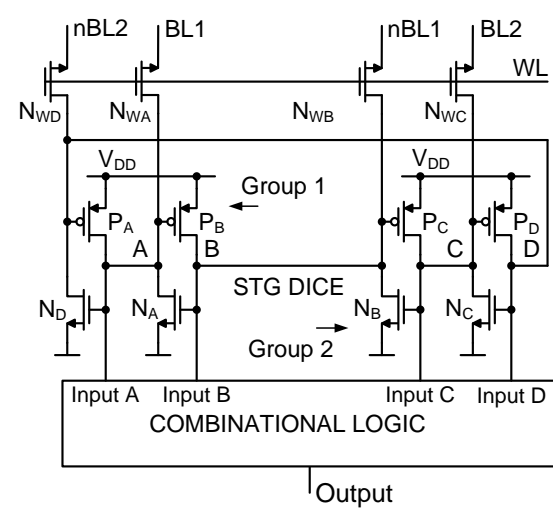

(a)

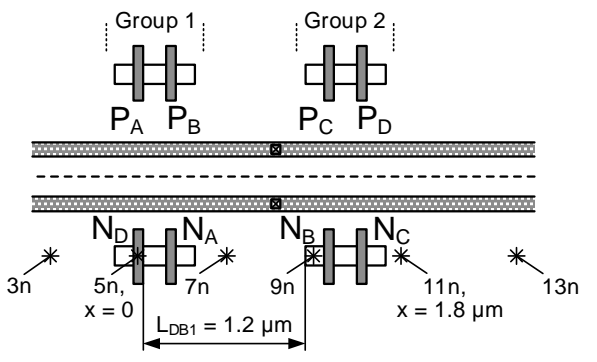

(b)

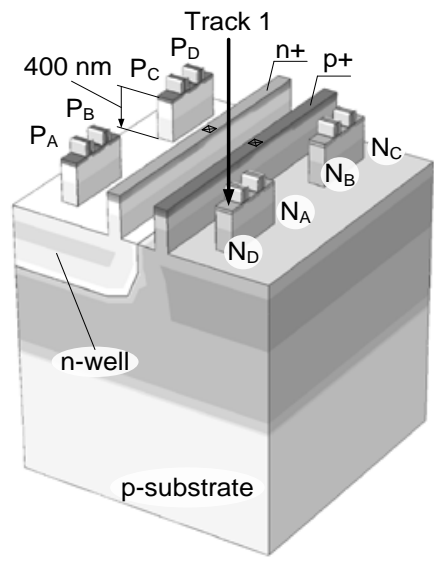

(c)

Fig. 1. STG DICE memory cell with the output combinational logic: (a) the scheme of the memory cell with transistors separated into two groups with spacing in between groups; (b) the layout of the topology of the cell, asterisks indicate the location of the input points of the particle tracks; (c) 3D TCAD device physics model of the cell. Tracks are the normal to the surface (as Track 1). The distance between transistors $N_{D}$ and $N_{B}$ is $1.2 \mu \mathrm{m}$

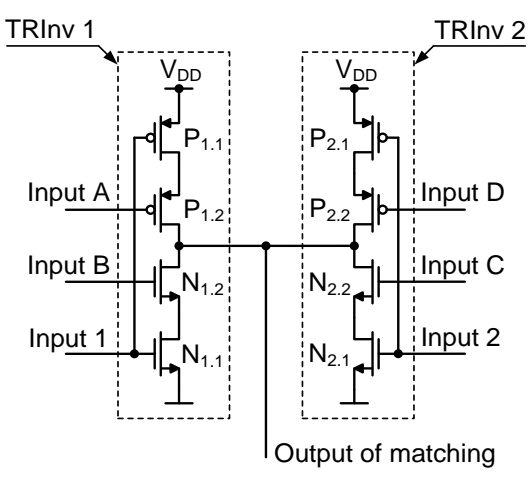

(a)

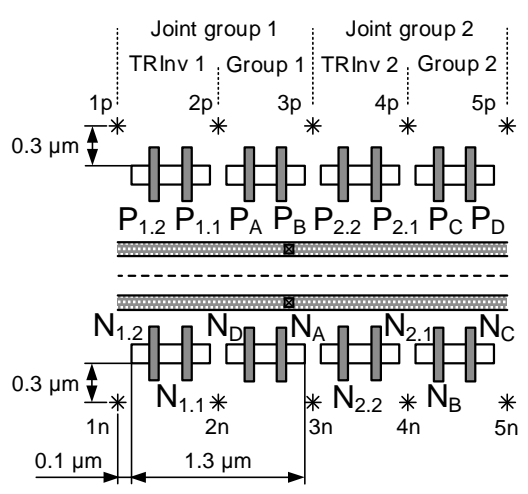

(b)

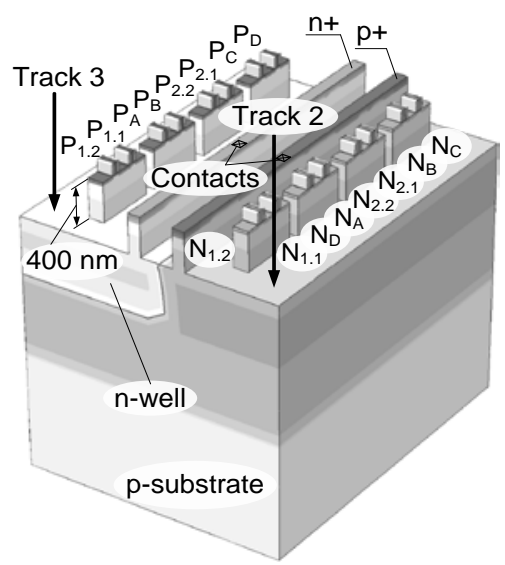

(c)

Fig. 2. The element of matching on STG DICE: (a) the scheme of the combinational logic of "Exclusive OR" on two tristate inverters TRInv 1 and TRInv 2; (b) the layout of the topology of the element; (c) 3D TCAD device physics model of the element. The distance between transistors $\mathrm{ND}_{\mathrm{D}}$ and $\mathrm{N}_{\mathrm{B}}$ of STG DICE cell is $1.35 \mu \mathrm{m}$. Asterisks in fig. $2 \mathrm{~b}$ indicate the location of the input point of the track

\section{SCHEMES OF ELEMENTS, LAYOUTS OF TOPOLOGIES, STRUCTURES OF DEVICE PARTS OF MODELS}

\section{A. STG DICE cell}

A scheme of STG DICE cell with a block of combinational logic is presented in Fig. 1a. The memory cell consists of four complementary pairs of CMOS transistors with each pair being the simple latch. Pairs $\mathrm{N}_{\mathrm{D}} \mathrm{P}_{\mathrm{A}}$ and $\mathrm{N}_{A} \mathrm{P}_{B}$ form the first group of transistors (Group 1), while the pairs $\mathrm{N}_{\mathrm{B}} \mathrm{P}_{\mathrm{C}}$ and $\mathrm{N}_{\mathrm{C}} \mathrm{P}_{\mathrm{D}}$ form the second group (Group 2). In the steady state, these pairs form a ring with interleaving pairs of open and closed transistors. For the logic of nodes $\mathrm{ABCD}=0101$, pairs $\mathrm{N}_{\mathrm{D}} \mathrm{P}_{\mathrm{A}}, \mathrm{N}_{\mathrm{B}} \mathrm{P}_{\mathrm{C}}$ are closed and pairs $\mathrm{N}_{A} \mathrm{P}_{B}, \mathrm{~N}_{C} \mathrm{P}_{\mathrm{D}}$ are open. The states of transistors change for the logic $\mathrm{ABCD}=1010$.

Fig. 1b presents a layout of STG DICE cell whose transistors are combined into two similar groups. The distance between closed transistors of the same conductivity type for both groups is $1.2 \mu \mathrm{m}$. The asterisks indicate the location of the input track points of particles in direction along the normal to the surface of the chip (the widths of all transistors are $150 \mathrm{~nm}$ ).

The 3D device physics structure for the STG DICE cell is depicted in Fig. 1c [12]. Device simulation of 65$\mathrm{nm}$ bulk CMOS transistors (with channels $65 \mathrm{~nm}$ length) was carried out using the 3D TCAD models of transistors presented in [13]. All tracks of nuclear particles are along the normal to the surface of the chip in p-region (as Track 1 in Fig. 1c). The oxide isolation of transistors (400 nm depth) is removed in Fig. 1c to make the image of the model better visible.

\section{B. Element of matching on STG DICE cell}

Fig. 2a shows a scheme of the combinational part of an element of matching on STG DICE cell as a XOR element on two tristate inverters TRInv 1 and TRInv 2. Fig. $2 b$ presents the layout of a topology for two groups. 


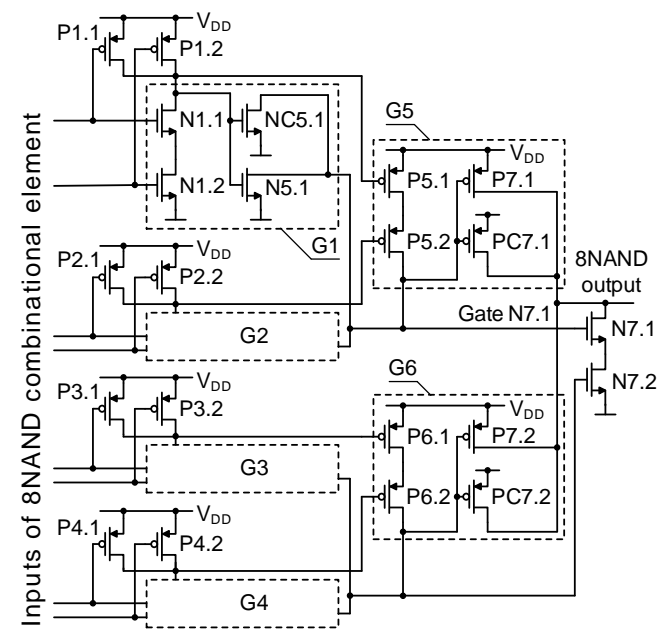

(a)

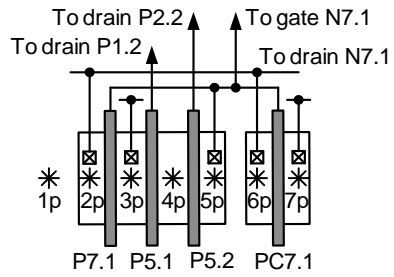

(c)

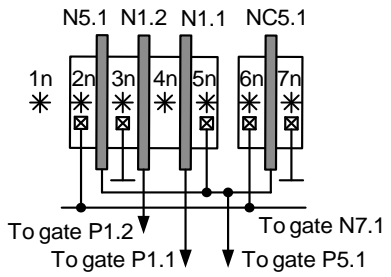

(b)

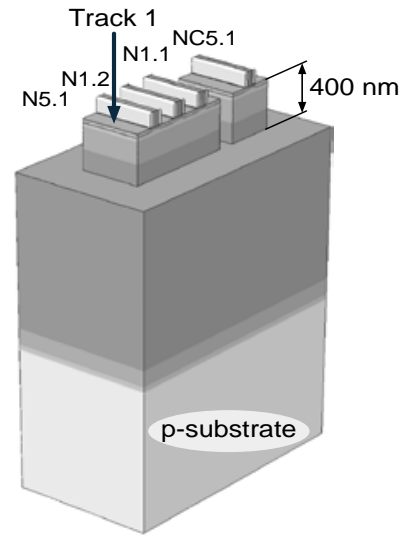

(d)

Fig. 3. The element of 8NAND on transistor groups G1-G6 with using additional transistors for the compensation of single event transients: (a) the scheme of the 8NAND element; (b) the layout of the group G1; (c) the layout of the topology of the group G5; (d) 3-D device physics model of one group G1, the direction of Track 1 is along the normal to the surface of the chip; asterisks in figs. $4 \mathrm{~b}, \mathrm{c}$ indicate the location of the input points of the particle tracks

The distance between transistor drains of two groups of STG DICE cell is $\mathrm{D}_{\mathrm{MIN}}=\mathrm{D}_{\mathrm{DB}}=1.35 \mu \mathrm{m}$. The asterisks indicate the location of the track input points during charge collection via the NMOS transistors (track points are $1 n-5 n$ ) and PMOS (track points are 1p-5p).

The 3D TCAD device physics model of the element of matching is presented in Fig. 2c [14]. The transistors correspond with two transistor groups (Group 1 or Group 2) in the STG DICE cell (see Figs. 1a and 1c) and with two tristate inverters TRInv 1 and TRInv 2 in Fig. 2a (widths of transistors are $150 \mathrm{~nm}$.

\section{Summing circuit based on combinational logic}

Summing circuits form the output signal of matching for whole row of output signals of matching and masking. In practice, summing circuits are designed on the base asynchronous combinational logic or synchronous circuits with wire match lines and their combinations. Combinational logic has lower speed, larger area and greater power consumption but is reliable. Fig. 3a presents the summing circuit based on logical elements NAND and NOR. The basic 8NAND element in Fig. 3a consists of five standard NAND gates (the first numbers of the transistors are the numbers of gates $1-4,7$ ) and two NOR gates (numbers 5 , 6 ). For example, the scheme of NAND gate consists of transistors P1.1, P1.2, N1.1 and N1.2.

An error pulse can change the output signal of mismatching to a false signal of matching on the output of the block of the summing logic. To avoid this, you must use a topology with the near location of the transistors for collecting charges from a track together in order to compensate error pulses. Such joint groups involve transistors of the same type from the neighboring logical gates. Fig. 3b presents the mutual layout of NMOS transistors in the group G1 and the location of the track input points. This preserves the correct summation result as the signal of mismatching at the output of the summing circuit.

The 8NAND element includes the six additional transistors NC5.1, NC5.2, NC5.3, NC5.2, PC7.1, PC7.2 for effective compensation of single event transients (SET) under impacts of single nuclear particles. The transistors of 8NAND element are combinated to six groups G1-G6, which includes the three transistors of the adjacent logical gates and the one additional compensation transistor. For example in the group G1, there are transistors N1.1, N1.2, N5.1 and the additional compensation transistor NC5.1. Each group contains the transistors, which have to be positioned jointly.

Fig. 3b shows the mutual layout of NMOS transistors in the group G1 and the location of the seven track input points. The asterisks indicate the track input points. The input track points $2 n, 4 n, 5 n, 6 n$ are the points in the drains of the transistors N5.1, N1.2, N1.1 and NC5.1. The input track point $3 \mathrm{n}$ is into the source of the transistors N5.1 and N1.2 (Fig. 3a). The track point $7 \mathrm{n}$ is into the source of the transistor NC5.1. The spacing in between the compensation transistor NC5.1 and the transistor N1.1 is the minimum distance $130 \mathrm{~nm}$ according the technological rule. Fig. 3c presents the mutual layout of PMOS transistors in the group G5 and the location of the track input points.

Fig. 3d shows the 3-D device physics model of one typical group G1 on NMOS transistors N1.1, N1.2, N5.1 and NC5.1. The device models of the groups G2-G4 are similar. The direction of Track 1 is along to the normal to the surface of the chip. The device physics model of the group G5 looks like the device model of the group G1. The length of the transistor channels is $60 \mathrm{~nm}$, the widths of NMOS transistors are $150-450 \mathrm{~nm}$ and the widths of PMOS transistors are 400-600 nm. 


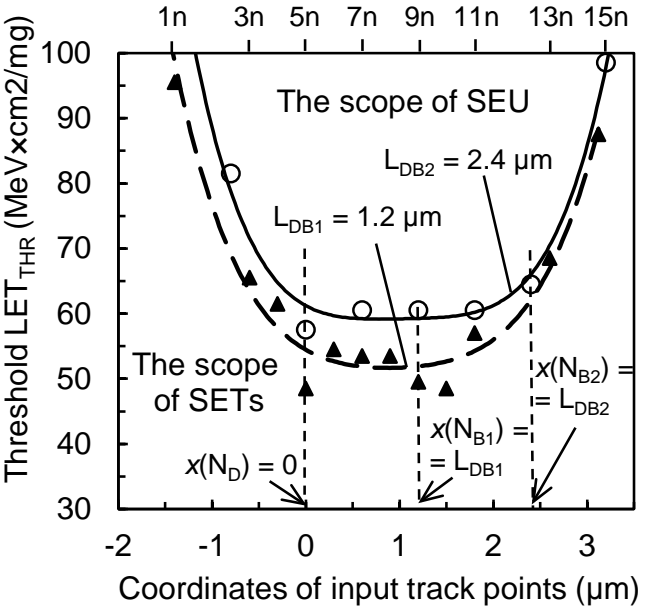

(a)

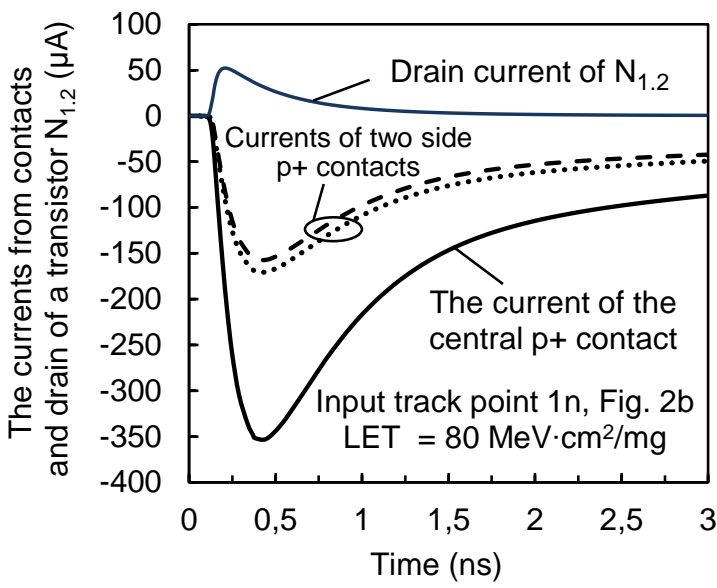

(b)

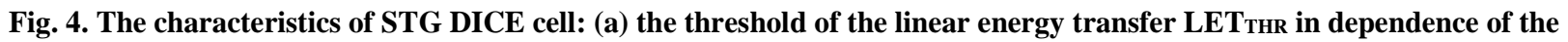
coordinate of the input track point; (b) the currents through the central and the two side $\mathbf{p}+$ contacts of the p+ guard band and the drain current of the transistor $\mathrm{N}_{1.2}$ on output of the combinational logic of the element of matching in Fig. $2 \mathrm{~b}$

\section{MAIN RESULTS OF SIMULATION}

The dependences for two different arrangements of transistor groups of the STG DICE cell are presented in Fig. 4a for the cases, when the distances between the drains of the transistors $\mathrm{N}_{\mathrm{D}}$ and $\mathrm{N}_{\mathrm{B}}$ are $\mathrm{L}_{\mathrm{DB} 1}=1.2 \mu \mathrm{m}$ and $\mathrm{L}_{\mathrm{DB} 2}=2.4 \mu \mathrm{m}$. Each point of the dependencies in Fig. $4 \mathrm{a}$ corresponds to the threshold values of a linear energy transfer (LET) that leads to an SEU when the track enters the chip through the point with the corresponding coordinate along the normal to the surface. The results are obtained during 3-D TCAD simulations using Sentaurus Device simulator at $25^{\circ} \mathrm{C}$ and the supply voltage of $1.0 \mathrm{~V}$ for $65 \mathrm{~nm}$ bulk CMOS structure [12].

Single event upsets (SEU) do not occur for the linear energy transfer below the threshold $\mathrm{LET}_{\mathrm{THR}}$. According to the simulation, in Fig. 4a the minimum threshold values of LET exceed the following for two distances: LET $_{\text {THR1.MIN }}$ $=49.5-53 \mathrm{MeV} \cdot \mathrm{cm}^{2} / \mathrm{mg}$ and $\mathrm{LET}_{\mathrm{THR} 2 \text { MIN }}=61-$ $63 \mathrm{MeV} \cdot \mathrm{cm}^{2} / \mathrm{mg}$. This correlates with a case of an ion ${ }^{136} \mathrm{Xe}$ impact with the energy $435 \mathrm{MeV}$, traversing a chip at normal incidence. The loss of the energy is near 30$32 \mathrm{MeV}$.

The result of the simulation of charges outputted through ohmic contacts of guard bands of the 3D models (models in Figs. 1c, 2c) and transistors of the combinational logic of the element of matching in dependence of the time during the charge collection is presented in Fig. 4b. The track along normal to the crystal surface, input track point - 1n (see Fig. 2b), LET = $80 \mathrm{MeV} \cdot \mathrm{cm}^{2} / \mathrm{mg}$, the initial state of the STG DICE cell is $\mathrm{ABCD}=1010$. The amplitudes of the current pulses through the central and the two side $\mathrm{p}^{+}$contacts of the $\mathrm{p}^{+}$ guard band significantly (5-10 times) exceed the amplitude of the current of the reverse biased pn junctions of the transistors that collected the charge in the combinational logic or the STG DICE cell from the same track.
The collected charges outputted through ohmic contacts of guard bands significantly (20-80 times) exceed the charge, which is collected through the pn junction of the transistor. Fig. $4 \mathrm{~b}$ presents the drain current of the transistor $\mathrm{N}_{1.2}$ as the part of the output combinational logic of the element of matching (the transistor $\mathrm{N}_{1.2}$ is in Fig. $2 \mathrm{~b}$ near the input track point $1 \mathrm{n}$ ) in time of the joint charge collection with ohmic contacts of bands [14].

Fig. 5a shows the dependencies of the amplitude and duration for an error output pulse of the element of matching for linear energy transfer to a track at LET = $60 \mathrm{MeV} \cdot \mathrm{cm}^{2} / \mathrm{mg}$. The curves show the error pulse parameters as functions of the coordinates of the track input points in case of matching the logical zero " 0 " stored in the STG DICE cell with the bit at Input $1=$ " 0 " (Input 2 = "1") at the combinational logic (Fig. 2a). The maximum amplitude of the error pulse is $1.3 \mathrm{~V}$ (track $1 \mathrm{n}$ ) for the pulse duration of $0.6 \mathrm{~ns}$. The amplitudes more $0.6 \mathrm{~V}$ with track input points $1 \mathrm{n}-3 \mathrm{n}$ correspond to logical error pulses.

At offset from the track input point $1 n$ to $5 n$ (Fig. 5a) the amplitude and the duration of an error impulse decrease monotonically and synchronously from their maximum to zero. Cases when an output bit of matching equals " 1 " are sensitive to noise pulses generated at the output of XOR logical element (error amplitude up to 0.8$1.3 \mathrm{~V}$ ). In other case, when the comparison bits is mismatching and the output logic equals " 0 " amplitudes of error pulses are less $0.3 \mathrm{~V}$.

Fig. 5b shows the amplitude and the duration of the output error pulse as dependence on the linear energy transfer (LET) to the track for two modes of the element of matching. The first mode is nodes ABCD $=0101$, Input 1 $=$ " 0 " (the white markers for the input track point $1 \mathrm{n}$ ) and the second mode is nodes $\mathrm{ABCD}=1010$, Input $1=$ " 1 " (the black markers for the input track point $3 n$ ). Up to $60 \mathrm{MeV} \cdot \mathrm{cm}^{2} / \mathrm{mg}$ dependences are close to linear functions 


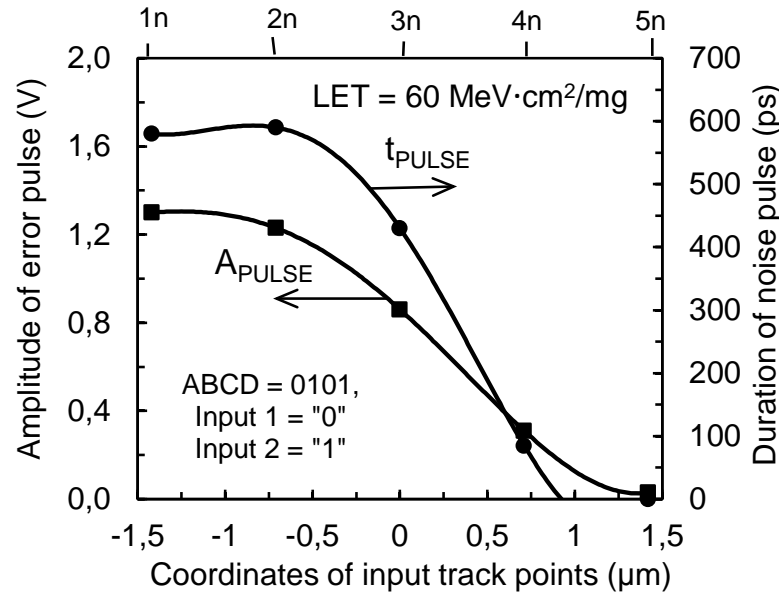

(a)

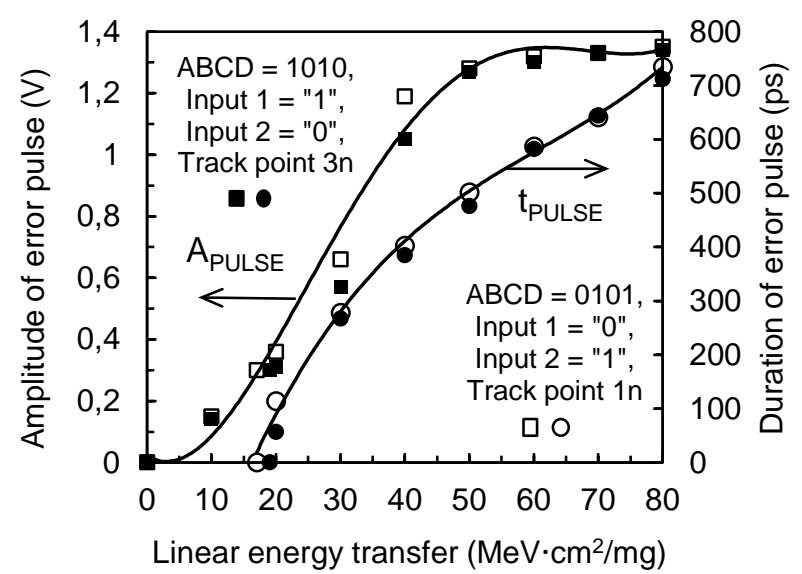

(b)

Fig. 5. The amplitude and the duration of the error pulse on the output of the combinational logic of the element of matching: (a) the functions of the input track point, the initial state of the STG DICE is ABCD = 0101, Input 1 is “0”, LET = $60 \mathrm{MeV} \cdot \mathrm{cm}^{2} / \mathrm{mg}$; (b) the functions of the linear energy transfer for two states of the element: 1 ) ABCD = 0101, Input $1=$ " 0 ", input track point is $1 \mathrm{n} ; 2) \mathrm{ABCD}=1010$, Input $1=$ “ 1 ", input track point is $3 \mathrm{n}$

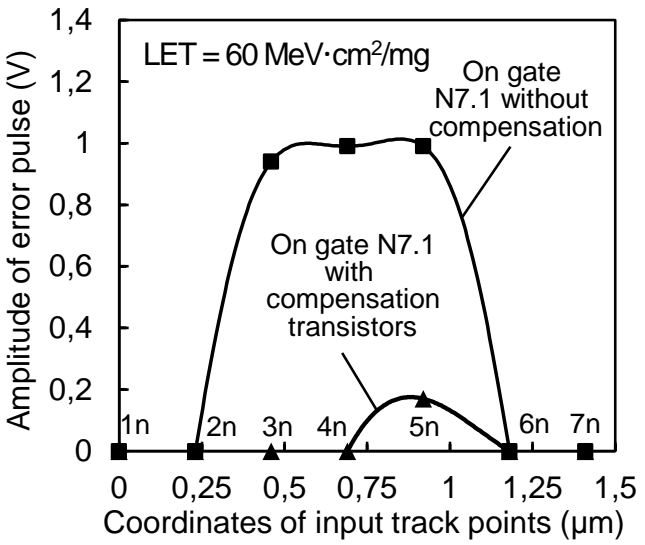

(a)

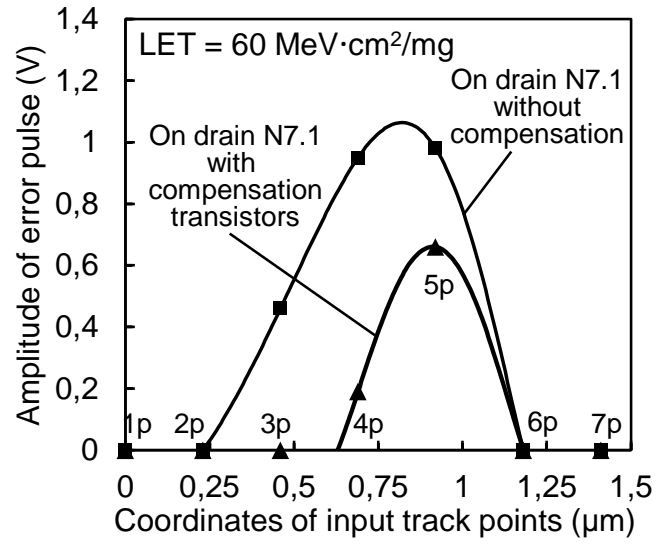

(b)

Fig. 6. Amplitudes of error pulses at NMOS transistor N7.1 of the 8NAND element with the compensation transistors or without them as functions of the input track points: (a) in the group G1, error pulse on gate N7.1; (b) in the group G5, error pulse on drain N7.1 (the output of the 8NAND element)

with the factor $0.2-0.25 \mathrm{~V} /\left(\mathrm{MeV} \cdot \mathrm{m}^{2} / \mathrm{mg}\right)$ of an increase in the amplitude of the error pulse and with the factor $10 \mathrm{ps} /\left(\mathrm{MeV} \cdot \mathrm{cm}^{2} / \mathrm{mg}\right)$ of an increase in the duration of the error pulse.

Figs. $6 \mathrm{a}$ and $6 \mathrm{~b}$ show the dependences, which illustrate the compensation of the error pulses acting at the gate or the drain of the output transistor N7.1 of the summation block on Fig. 3a. Fig. 6a shows the dependences of the amplitudes of the error pulse at the gate of the NMOS transistor N7.1. This dependence is the function of the displacement of the input track points from $1 \mathrm{n}$ up to $7 \mathrm{n}$ in the group of transistors G1 (Fig. 3b) along the line of the drains of transistors N5.1, N1.2, N1.1 and NC5.1. The LET on the track was $60 \mathrm{MeV} \cdot \mathrm{cm}^{2} / \mathrm{mg}$.

The amplitudes of the error pulses equal nil for the input track points $1 \mathrm{n}, 2 \mathrm{n}$ and $6 \mathrm{n}, 7 \mathrm{n}$ in cases with the com- pensation transistor or without. The amplitudes of the error pulses without compensation (Fig. 6a) are practically unchangeable and equal $1 \mathrm{~V}$ for the input track points $3 \mathrm{n}$, $4 n$ and $5 n$. This shows that the output of the summation block will be nil, which means a false signal of matching at the output. In contrast, when the compensation is used, the amplitude of the noise pulse is $0.17 \mathrm{~V}$ for the point $5 \mathrm{n}$ and equals nil for the other input track points (Fig. 6a) That means the right signals of mismatching at the output.

Fig. $6 \mathrm{~b}$ shows the amplitudes of the error pulses at the drain of the output transistor N7.1 (on the output of the 8NAND element) when the input track point the input track point is displaced from $1 p$ up to $7 p$ in the group G5 (Fig. 3c). It is the line along the drains of transistors P7.1, P5.1, P5.2 and PC7.1. The LET on the track was $60 \mathrm{MeV} \times \mathrm{cm}^{2} / \mathrm{mg}$. In case of no compensation (Fig. 6b), amplitudes of error pulses are in the range $0.4-1.0 \mathrm{~V}$ for 
the input track points $3 p, 4 p$ and $5 p$. In contrast, when the compensation is used the amplitudes of the noise pulses are $0.4-0.65 \mathrm{~V}$ for the points $4 \mathrm{p}$ and $5 \mathrm{p}$ and equal nil for the other input track points. That means the right signals of mismatching at the output with the compensation.

The error pulse of the group G1 acts on the gate of the closed transistor N1.7, and the error pulse of the group G5 acts on the 8NAND output. In both cases, these error pulses are compensated up to amplitudes (Figs. 9, 10) that do not allow changing the logical state of mismatching on the output the summing element.

\section{DISCUSSION}

The STG DICE cell $65 \mathrm{~nm}$ bulk CMOS technology reliably stores the data written therein up to LET = $60 \mathrm{MeV} \cdot \mathrm{cm}^{2} / \mathrm{mg}$. The element of matching (Fig. 2) is the main element of an associative memory.

For the element of matching with the Output $=$ " 1 " the maximum of amplitudes of error pulses at the output of combinational part occurs when the charge is collected from the tracks passing near the closed NMOS transistors of the inverter TRInv 1 or TRInv 2 (Fig. 2b). This result of the simulation is illustrated by the amplitudes and the durations of the noise pulses with the input track points $1 \mathrm{n}$ to $4 \mathrm{n}$ on Fig. 5a.

The main result of simulation of the charge collection by NMOS transistors in the states of STG DICE nodes $\mathrm{ABCD}=0101$ for Input $1=$ " 1 " and $\mathrm{ABCD}=1010$ for Input $1=$ " 0 " is that the charge collection does not affect the logic level of the output bit " 0 " in this state of mismatching of the data. This conclusion is based on the fact that the noise pulses at the output of the element does not exceed $0.26 \mathrm{~V}$ up to LET $=60 \mathrm{MeV} \cdot \mathrm{cm}^{2} / \mathrm{mg}$.

The main result of simulation of the charge collection by PMOS transistors in the states of STG DICE nodes $\mathrm{ABCD}=0101$ and $\mathrm{ABCD}=1010$ for both Input $1=$ " 0 " and Input $1=$ " 1 " is that the charge collection does not affect the logic level of the output bit. In these cases, the noise pulses at the output of the element of matching does not exceed $0.17 \mathrm{~V}$ up to $\mathrm{LET}=60 \mathrm{MeV} \cdot \mathrm{cm}^{2} / \mathrm{mg}$.

\section{CONCLUSIONS}

The analysis of charge collecting from tracks of single nuclear particles based on the TCAD simulation allows you to get the results of research that is not available by experimental methods. The results of the TCAD simulations of transient processes under impacts of single nuclear particles on the new elements of an associative memory show that 65-nm cells STG DICE reliably store the logical state at the linear energy transfer up to $60 \mathrm{MeV} \cdot \mathrm{cm}^{2} / \mathrm{mg}$. The same results are for the new elements of matching and masking based on STG DICE cells.

The important part of failure tolerance of elements of matching and masking is the effects at the outputs of the combinational logic of these elements. Pulses of logical errors in the output logic can be only in elements of matching up to duration $0.3-0.6 \mathrm{~ns}$ in the range of the energy transfer 30-60 $\mathrm{MeV} \cdot \mathrm{cm}^{2} / \mathrm{mg}$.

The single event transients in elements of summing are minimized by separation transistors into special groups and co-location the compensation transistors in each group. The elements on the STG DICE cells make it possible to improve the hardness of associative memories to impacts of ionizing particles.

\section{REFERENCES}

[1] Pagiamtzis K., Sheikholeslami A. Content-addressable memory (CAM) circuits and architectures: a tutorial and survey. IEEE Journal of Solid-State Circuis, 2006, vol. 41, no. 3, pp. 712-727.

[2] Annovi A., Calderini G., Crescioli F., De Canio F., Frontini L., Kubota T., Liberali V., Luciano P., Palla F., Shojaii S. R., Sotiropoulou C-L., Stabile A., Traversi G. A lowpower and high-density associative memory in $28 \mathrm{~nm}$ CMOS Technology. Proceedings of International Conference on Modern Circuits and Systems Technologies (MOCAST), 2017, pp. 1-4.

[3] Frontini L., Shojaii S., Stabile A., Liberali V. A new XORbased content addressable memory architecture. Proceedings of IEEE International Conference on Electronics, (ICECS), 2012, pp. 701-704.

[4] Abbas S. M., Lee S., Baeg S., and Park S. An efficient multiple cell upsets tolerant content-addressable memory. IEEE Transactions on Computers, 2014, vol. 63, no. 8, pp. 2094-2098.

[5] Azizi N., Najm F. A family of cells to reduce the soft-errorrate in ternary-CAM. Proceedings of Design Automation Conference, 2006, pp. 779-784.

[6] Eftaxiopoulos N., Axelos N., Pekmestzi K. Low leakage radiation tolerant CAM/TCAM cell. IEEE International On-Line Testing Symposium (IOLTS), 2015, pp. 206-211.

[7] Katunin Yu. V., Stenin V. Ya., Stepanov P. V. Modeling the characteristics of trigger elements of two-phase CMOS logic, taking into account the charge sharing effect under exposure to single nuclear particles. Russian Microelectronics, 2014, vol. 43, no. 2, pp. 112-124.

[8] Stenin V.Ya. Simulation of the characteristics of the DICE 28-nm CMOS cells in unsteady states caused by the effect of single nuclear particles. Russian Microelectronics, 2015, vol.. 44, no. 5, pp. 324-334.

[9] Calin T., Nicolaidis M., Velazco R. Upset hardened memory design for submicron CMOS technology. IEEE Transactions on Nuclear Science, 1996, vol. 43, no. 6, pp. 2874-2878.

[10] Stenin V.Ya., Stepanov P.V. Basic memory elements using DICE cells for fault-tolerant $28 \mathrm{~nm}$ CMOS RAM. Russian Microelectronics, 2015, vol. 44, no. 6, pp. 368-379.

[11] Stenin V.Ya., Katunin Yu.V., Stepanov P.V. Upset-resilient RAM on STG DICE memory elements with the spaced transistors into two groups. Russian Microelectronics, 2016, vol. 45, no. 6, pp. 419-432.

[12] Katunin Yu.V., Stenin V.Ya. Simulation of single event effects in STG DICE memory cells. Russian Microelectronics, 2018, vol. 47, no. 1, pp. 20-33.

[13] Garg R., Khatri S.P. Analysis and design of resilient VLSI circuits: mitigating soft errors and process variations. New York: Springer, 2010. pp. 194-205.

[14] Katunin Yu.V., and Stenin V.Ya. The element of matching on an STG DICE Cell for an Upset Tolerant content addressable memory. Russian Microelectronics, 2018, vol. 47, no. 2, pp. 142-156. 